\title{
REMARK ON A CONJECTURE OF KAPLAN AND YORKE
}

\author{
ANGELIKA WÖRZ-BUSEKROS
}

\begin{abstract}
If all solutions of a system of quadratic differential equations with at least one stationary solution are bounded, then the corresponding homogeneous equation possesses a line of stationary points.
\end{abstract}

The purpose of this note is to show that a conjecture of Kaplan and Yorke on the relationship between the behavior of solutions of a system of quadratic differential equations and of the associated homogeneous system is true.

Let us consider a system of nonlinear quadratic differential equations in $\mathbf{R}^{n}$ which possesses at least one stationary solution $x^{*}$. After a preliminary translation $x^{*} \mapsto 0$ the system can be written as

$$
\dot{x}=A x+f(x), \quad x \in \mathbf{R}^{n},
$$

where $A$ is a real $n \times n$-matrix, every component of $f$ either vanishes or is homogeneous of degree two in the coordinates of $x$, and there is at least one nonvanishing component. Besides (1) we consider the associated homogeneous equation

$$
\dot{x}=f(x), \quad x \in \mathbf{R}^{n} .
$$

Kaplan and Yorke [3] conjectured the following relationship between solutions of (1) and (2) which turns out to be true.

THEOREM 1. If all solutions of (1) are bounded, then the differential equation (2) possesses a line through the origin consisting of stationary points.

The proof of this theorem is based on a sequence of theorems of Markus [4], Dickson and Perko [1, 2] and Kaplan and Yorke [3]. In order to present some results of Markus let us first indicate several relationships between solutions of (2) and an algebra $\mathfrak{Q}$ associated with (2). Let $f=\left(f_{1}, \ldots, f_{n}\right)^{T}$ with

$$
f_{k}(x)=\sum_{i, j=1}^{n} x_{i} \gamma_{i j k} x_{j}, \quad k=1, \ldots, n,
$$

where we have distributed the parts of $x_{i} x_{j}$ and $x_{j} x_{i}$ equally, i.e.

$$
\gamma_{i j k}=\gamma_{j i k} \quad \text { for all } i, j, k=1, \ldots, n \text {. }
$$

In the vector space $\mathbf{R}^{n}$ spanned by the unit vectors $e_{1}, \ldots, e_{n}$ we introduce a multiplication by

$$
e_{i} e_{j}:=\sum_{k=1}^{n} \gamma_{i j k} e_{k}, \quad i, j=1, \ldots, n,
$$

Received by the editors September 14, 1981.

1980 Mathematics Subject Classification. Primary 34C05.

Key words and phrases. Inhomogeneous and homogeneous quadratic differential equations.

(C) 1982 American Mathematical Society 0002-9939/81/0000-0262/\$02.00 
and its bilinear extension onto $\mathbf{R}^{n} \times \mathbf{R}^{n}$. Thereby $\mathbf{R}^{n}$ gets an algebra $\mathcal{U}$ which is commutative in view of (3) but, in general, not associative. In terms of the algebra multiplication the differential equation (2) can be written as

$$
\dot{x}=x^{2}, \quad x \in \mathscr{U} \text {. }
$$

An element $0 \neq e \in \mathscr{U}$ is called an idempotent if $e^{2}=e$. Furthermore an element $0 \neq n \in \mathfrak{l}$ is called a nilpotent element of index 2 if $n^{2}=0$. The following two basic results on the relationship between the algebra $\mathcal{Q}$ and the differential equation (2) have been proved by Markus [4].

THEOREM 2. The origin is an isolated critical point of (2) if and only if $\mathcal{U}$ does not possess a nilpotent element of index 2 .

In other words, the origin is not an isolated rest point if and only if $थ$ possesses a nilpotent element. In this case the straight line joining the origin and this nilpotent element consists of stationary points, cf. Markus [4].

THEOREM 3. The differential equation (2) has a ray solution if and only if 2 t possesses an idempotent.

The following theorem is due to Markus [4] for odd $n$ and to Dickson and Perko [1, Theorem 1, §3], as well as Kaplan and Yorke [3] for arbitrary $n$. Dickson and Perko's formulation reads

THEOREM 4. A ray solution of (2) exists if the origin is an isolated rest point.

Furthermore we need the following fundamental result of Dickson and Perko [1, Theorem 2, §3].

THEOREM 5. If (2) has a ray solution, then the system (1) has an unbounded solution of finite escape time.

Now Theorem 1 follows by the negative versions of Theorems 5, 4 and 2 and the remark subsequent to Theorem 2 .

Finally let us remark that the boundedness of solutions of (1) does not imply the boundedness of solutions of (2) as it was pointed out by Kaplan and Yorke [3], referring to examples of Dickson and Perko [2]. These examples are based on the fact that for $n=2$ the differential equation (2) may have unbounded solutions even if it has no ray solution and, in addition, there exists a class of operators $A$ such that all solutions of (1) are bounded.

\section{REFERENCES}

1. R. J. Dickson and L. M. Perko, Quadratic differential systems, Lockheed Research Report No. LMSC/L-56-68-1, Lockheed Palo Alto Research Lab., February 1968.

2. - Bounded quadratic systems in the plane, J. Differential Equations 7 (1970), 251-273.

3. J. L. Kaplan and J. A. Yorke, Nonassociative, real algebras and quadratic differential equations, Nonlinear Anal., Theory, Methods and Appl. 3 (1979), 49-51.

4. L. Markus, Quadratic differential equations and nonassociative algebras, Contributions to the Theory of Nonlinear Oscillations (L. Cesari, J. LaSalle, S. Lefschetz (eds.)), Princeton Univ. Press, Princeton, N.J., 1960, pp. 185-213.

Lehrstuhl Für BiOMATHematik, AUf DeR MORgenstelle 28, 7400 TüBiNgeN 1, FEDERAL REPUBLIC OF GERMANY 